\title{
Research on the Working Mechanism of Melt Metallurgical Effect
}

\author{
Liao Xiaoyang $^{1^{*}}$, Zhuang Jinwu $^{1}$, Liu Yazhou ${ }^{1}$ \\ ${ }^{1}$ School of Electrical Engineering, Naval University of Engineering, Wuhan, Hubei, China
}

\begin{abstract}
This paper takes rectangular tinned silver flake as the research object to explore the working mechanism of melt metallurgical effect. Firstly, the paper designs and processes rectangular tinned silver flake through the electroplating technology, then conducts low-overload pre-arc experiment with the metallurgical samples. According to the experiment results, it is proposed that "Under low overload current, the formation of silver-tin alloy leads to the increase of current density of the metallic silver layer, which significantly advances the break time of the silver flake. At the same time, the silver-tin interpenetration speed is a linear function of the temperature." Finally, the self-programming software is used to establish the transient electric heating field model of the metallurgical tinned silver flake. By comparing simulation and experiment, the results verify the accuracy of the hypothesis, and obtain the working mechanism of the melt metallurgical effect, which provides a reliable theoretical basis for the design and application of the metallurgical trigger.
\end{abstract}

\section{Introduction}

The metallurgical effect refers to when the flux with a low melting point, which places on the surface of the melt, reaches its own melting point, it would melt, penetrate and form alloy with the melt. Zhou Ting proposed that once the fuse has a current overload phenomenon, a diffusion process will occur on the soft tin of the melt, which leads to the continuous increase of melt resistance, accelerates the melting of soft tin solder joint and finally breaks the circuit [1]. Fu Xue used tinned copper flake to conduct metallurgical experiments and found that the metallurgical effect effectively improves the low-overload breaking capacity of the melt. At the same time, she proposed that the metallurgical effect is a dynamic alloy formation process and the alloy formation speed is a constant [2]. However, the current research on metallurgical effects is still in the exploratory stage, and its complete working mechanism and theoretical research are not perfect.

${ }^{1}$ In order to explore the working mechanism of melt metallurgical effect, this paper takes rectangular tinned silver flake as the research object. Firstly, the paper processes metallurgical samples, then conducts metallurgical pre-arc experiments under low overload current [3], proposes reasonable assumptions about the working principle of metallurgical effects; Finally, the paper establishes an electrothermal coupling mathematical model of metallurgical tinned silver flake, and compares the simulation results with the experiment ones. The results are basically the same, so the paper obtains the working mechanism of the melt metallurgical effect, which provides a reliable theoretical basis for the subsequent design and processing of metallurgical arc trigger.

\section{Designing of metallurgical samples}

\subsection{Metallurgical sample design}

In this paper, a rectangular silver flake is selected as the melt, and tin layer of a certain thickness is plated on its upper surface as the flux [4]. After the sample is processed, it is welded on the left and right sides of the copper block by the electric welding machine [5]. In order to reduce the influence of the heat dissipation of the connected copper block on the pre-arc experiment results and ensure the accuracy of the electroplating thickness measurement, the size of the rectangular silver sample is $30 \times 10 \times 0.1 \mathrm{~mm}$, the flow cross-section's area unit of copper block is $90 \mathrm{~mm}^{2}$, and the thickness of the tin layer is $10 \mathrm{um}$.

\subsection{The calculation of Electroplating thickness}

When the current passes through the electrolyte, the thickness of the deposit on the cathode surface can be calculated according to Faraday's law [6]. It is shown as formula (1). This article chooses single-sided tin plating, and the thickness of the tin layer is $10 \mathrm{um}$.

$$
\sigma=\frac{100 I t \eta k}{S \rho}
$$

\section{Low-overload experiment of metallurgical tinned silver flake}

In order to explore the working mechanism of the

*Corresponding author: 2598923898@qq.com 
metallurgical effect, this paper builds a temperature rise experiment platform to conduct a low-overload pre-arc experiment with metallurgical tinned silver samples.

\subsection{Analysis of experimental results}

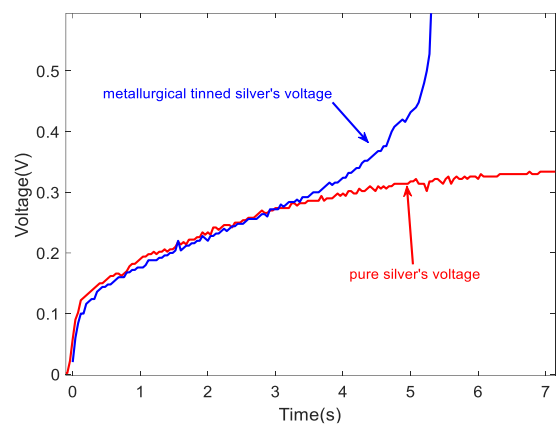

Figure 1. Voltage waveforms of metallurgical and pure silver samples

It can be seen from Figure 1 that when the 220A current is applied to the metallurgical sample and the pure silver sample, at $5.2 \mathrm{~s}$, the metallurgical sample breaks, generating arc voltage, and the voltage waveform rises sharply, while the pure silver flake's voltage waveform maintains a parallel trend, and refuses to break. Figure 1 also shows that the voltage waveforms of metallurgical and pure silver flake are basically the same before $2.8 \mathrm{~s}$. After $2.8 \mathrm{~s}$, the voltage curve of the former is always higher than the latter, indicating that the tin begins to melt and penetrates at this moment to form silver-tin alloy. Therefore, it can be known that under the condition of $220 \mathrm{~A}$ current, due to the metallurgical effect, the pre-arc time of the silver flake is greatly advanced, and the metallurgical effect time is about $2.4 \mathrm{~s}$.

\subsection{Speculation on the working mechanism of metallurgical effects}

In view of the experimental phenomenon, this paper makes the following speculations on the working mechanism of the metallurgical effect:

- When the temperature of the tin layer reaches the melting point temperature, the tin begins to melt, penetrate, and form silver-tin alloy. The three processes can be considered to be synchronized;

- The melting rate (that is the penetration rate of tin or the formation rate of silver-tin alloy) is a function of the temperature of the sample;

- After the current passes, the thickness of the silver layer is dynamically reduced, and the flow density is dynamically increased, which increases the temperature rise of the silver layer and accelerates the process of the silver layer reaching the melting point temperature $\left(960^{\circ} \mathrm{C}\right)$.

\section{The analysis of metallurgical silver flake's model}

\subsection{Geometric model}

Based on experimental results and reasonable speculations, this paper conducts modeling analysis on metallurgical tinned silver flake. As shown in Figure 2, the mathematical model is mainly divided into two parts. The first part is that before the tin layer melts, the metal tin layer and the pure silver layer are connected in parallel without any change in geometric thickness. As the schematic diagram of geometric model is shown in Figure 2 (b), when the highest temperature of the tin layer reaches its melting point temperature $\left(237^{\circ} \mathrm{C}\right)$, the second part starts. During this process, there are three kinds of metals in parallel, and there are thickness changes.

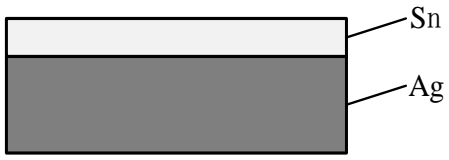

(a) The first stage of metallurgical effect

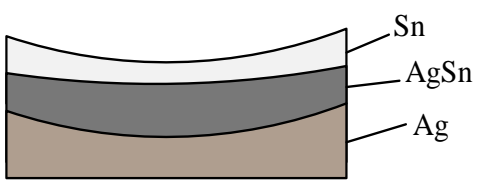

(b) The second stage of metallurgical effect

Figure 2. The schematic diagram of metallurgical effect

\subsection{Mathematical equation}

Considering that the shape of the sample is regular and the temperature in the thickness direction does not change much compared to the length direction, this paper establishes a one-dimensional mathematical model for the metallurgical silver flake along the current flow direction. The expression of the electrothermal field coupling equation is shown as formula (2).

$$
c m \frac{d T}{d t}=I^{2} R+V \lambda \frac{d^{2} T}{d x^{2}}
$$

\subsection{Identification of Mathematical Equation Parameters}

\subsubsection{Product of unit element's specific heat capacity and mass}

In the first and second stages, the formulas of product of unit element's specific heat capacity and mass are different, the expressions are shown as formula (3) and formula (4).

$$
\begin{aligned}
(\mathrm{Cm})_{\mathrm{i}} & =C_{\mathrm{Ag}} \rho_{\mathrm{Ag}} H_{\mathrm{Ag}} W h+C_{\mathrm{Sn}} \rho_{\mathrm{Sn}} H_{\mathrm{Sn}} W h \\
(\mathrm{Cm})_{\mathrm{i}}= & C_{\mathrm{Ag}} \rho_{\mathrm{Ag}} H_{\mathrm{i}(\mathrm{Sn})} W h+C_{\mathrm{Sn}} \rho_{\mathrm{Sn}} H_{\mathrm{i}(\mathrm{Sn})} W h \\
& +C_{\mathrm{AgSn}} \rho_{\mathrm{AgSn}} H_{\mathrm{i}(\mathrm{AgSn})} W h
\end{aligned}
$$

In the formula, $C_{\mathrm{Sn}}, C_{\mathrm{Ag}}, C_{\mathrm{AgSn}}$ are the specific heat capacities of the three materials. By consulting the 
information, take $220,240,230$, the unit is $\mathrm{J} \cdot\left(\mathrm{kg}^{\circ} \mathrm{C}\right)^{-1} ; \rho_{\mathrm{Ag}}$ is the density of silver, take $10530 \mathrm{~kg} \cdot\left(\mathrm{m}^{3}\right)^{-1} \cdot \rho_{\mathrm{Sn}}$ is the density of tin, which is $7280 \mathrm{~kg} \cdot\left(\mathrm{m}^{3}\right)^{-1}$. At the same time, the density of silver-tin alloy takes $13530 \mathrm{~kg} \cdot\left(\mathrm{m}^{3}\right)^{-1} ; W$ is the width of the sample, which is taken $10 \mathrm{~mm}$; $\mathrm{h}$ is the spatial step length. Since the grid is divided into 100 grids, $h$ is taken as $0.3 \mathrm{~mm}$; in the first stage, $H_{\mathrm{Ag}}$ and $H_{\mathrm{Sn}}$ are the initial thicknesses of the metal layer, which takes $0.1 \mathrm{~mm}$

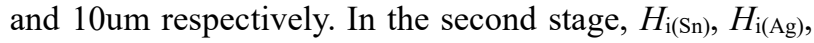
$H_{\mathrm{i}(\mathrm{AgSn})}$ are variables. Based on speculation, the calculation formula is shown as formula (5).

$$
\left\{\begin{array}{l}
H_{\mathrm{i}(\mathrm{Sn})}=H_{\mathrm{Sn}}-v\left(T_{\mathrm{i}}\right) t \\
H_{\mathrm{i}(\mathrm{Ag})}=H_{\mathrm{Ag}}-v\left(T_{\mathrm{i}}\right) t \\
H_{\mathrm{i}(\mathrm{AgSn})}=v\left(T_{\mathrm{i}}\right) t
\end{array}\right.
$$

In the formula, $t$ is the penetration time; $v\left(T_{\mathrm{i}}\right)$ is the penetration rate of the tin, which has a linear function relationship with the unit cell temperature $T_{\mathrm{i}}$. The formula is shown as formula (6), and the unit is $\mathrm{m} / \mathrm{s}$ :

$$
v=\left\{\begin{array}{c}
0 \quad T<237 \\
1.7 \mathrm{E}^{-8} \times(T-237) \quad T \geq 237
\end{array}\right.
$$

\subsubsection{Unit element's resistance}

At different stages, the parallel metal is different, and the unit element's resistance calculation formula is also different. The formulas are shown as formula (7) and formula (8).

$$
\begin{gathered}
\left\{\begin{array}{l}
R_{\mathrm{i}(\mathrm{Sn})}=r_{\mathrm{Sn}}^{0} 1+k_{\mathrm{Sn}}\left(T_{\mathrm{i}}-T_{0}\right) h\left(H_{\mathrm{Sn}} W\right)^{-1} \\
R_{\mathrm{i}(\mathrm{Ag})}=r_{\mathrm{Ag}}^{0} \mid 1-k_{\mathrm{Ag}}\left(T_{\mathrm{i}}-T_{0}\right) h\left(H_{\mathrm{Ag}} W\right)^{-1} \\
R_{\mathrm{i}}=R_{\mathrm{i}(\mathrm{Sn})} R_{\mathrm{i}(\mathrm{Ag})}\left(R_{\mathrm{i}(\mathrm{Sn})}+R_{\mathrm{i}(\mathrm{Ag})}\right)^{-1}
\end{array}\right. \\
\mid \begin{array}{ll}
R_{\mathrm{i}(\mathrm{Sn})}=r_{\mathrm{Sn}}^{0} 1 \mid k_{\mathrm{Sn}}\left(T_{\mathrm{i}} T_{0}\right) h\left(H_{\mathrm{i}(\mathrm{Sn})} W\right)^{-1} \\
R_{\mathrm{i}(\mathrm{Ag})}=r_{\mathrm{Ag}}^{0}|1| k_{\mathrm{Ag}}\left(T_{\mathrm{i}} \quad T_{0}\right) \mid h\left(H_{\mathrm{i}(\mathrm{Ag})} W\right)^{\cdot 1} \\
R_{\mathrm{i}(\mathrm{AgSn})}=r_{\mathrm{AgSn}}^{0}|1| k_{\mathrm{AgSn}}\left(T_{\mathrm{i}} T_{0}\right) \mid h\left(H_{\mathrm{i}(\mathrm{AgSn})} W\right)^{11} \\
R_{\mathrm{i}}=R_{\mathrm{i}(\mathrm{Sn})} R_{\mathrm{i}(\mathrm{Ag} g} R_{\mathrm{i}(\mathrm{AgSn})}\left(R_{\mathrm{i}(\mathrm{Sn})} R_{\mathrm{i}(\mathrm{Agg})}\left|R_{\mathrm{i}(\mathrm{Ag})} R_{\mathrm{i}(\mathrm{AgSn})}\right| R_{\mathrm{i}(\mathrm{Sn})} R_{\mathrm{i}(\mathrm{AgSn})}\right)^{-1}
\end{array}
\end{gathered}
$$

In the formula, $R_{\mathrm{i}(\mathrm{Sn})}, R_{\mathrm{i}(\mathrm{Ag})}, R_{\mathrm{i}(\mathrm{AgSn})}$ are the resistances of the tin layer, silver layer, and silver-tin alloy layer of unit element respectively, and $R_{\mathrm{i}}$ is the total resistance of the unit element; $r^{0} \mathrm{Sn}, r_{\mathrm{Ag}}^{0}, r_{\mathrm{AgSn}}^{0}$ are the resistivity of the three materials at $20^{\circ} \mathrm{C}$, which takes $7.042 \times 10^{-8}, 1.81 \times 10^{-}$ ${ }^{8}, 1.81 \times 10^{-7}$ respectively, and the unit is $\mathrm{S} / \mathrm{m} ; k_{\mathrm{Sn}}, k_{\mathrm{Ag}}$, and $k_{\mathrm{AgSn}}$ are the temperature coefficients of resistivity, taking $0.0047,0.0039,0.003$.

\subsubsection{Unit element's volume}

The formula for calculating the volume of the unit element in the first and second stages is shown in formulas (9) and formulas (10).

$$
\begin{array}{r}
V_{\mathrm{i}}=h W\left(H_{\mathrm{Sn}}+H_{A g}\right) \\
V_{\mathrm{i}}=h W\left(H_{\mathrm{i}(\mathrm{Ag})}+H_{\mathrm{i}(\mathrm{Sn})}+H_{\mathrm{i}(\mathrm{AgSn})}\right)
\end{array}
$$

\subsection{Comparative analysis of simulation and experiment results}

In this paper, under the low-overload 220A, the simulation and experiment pre-arc voltage waveform are compared and analyzed, as shown in Figure 3.

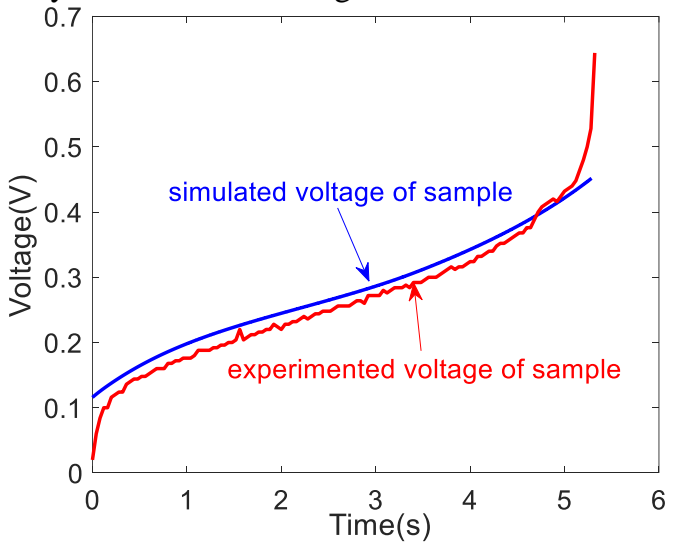

Figure 3. Comparison of the simulation and experiment voltage curves

According to the comparison of the waveforms between the experiment and simulation in Figure 3, it can be found that the waveform trends are slightly different at the beginning of the current flow and at the end of the breaking time. The reasons are analyzed in sequence: (1) In the experiment, the current is stepped up from the value 0 through the discharge of the capacitor, and after $10 \mathrm{~ms}$, The current is stable at $220 \mathrm{~A}$, and the current is set to 220 from time 0 in the simulation, so the experiment and simulation voltage waveforms cannot be completely consistent at the initial stage, but consider that the $10 \mathrm{~ms}$ step time is shorter than the pre-arc time, and the sample is tested in this process The temperature rise is not obvious so the error is acceptable; (2) In the experiment, the metallurgical sample generates an arc at all times, and the voltage waveform trend rises sharply under the action of the arc voltage. However, in the simulation, the highest temperature of the sample is set to reach the melting point The $960^{\circ} \mathrm{C}$ simulation ends without considering the influence of arc voltage, so the waveforms of the two are inconsistent at the moment of action, but this article focuses on the working mechanism of metallurgical effects without considering the size of the arc, so the inconsistency of the waveform in the later period can be ignored. Therefore, through the comparison of simulation and experimental voltage waveforms, it can be considered that the inference of the metallurgical effect working mechanism after the test is reasonable and correct, which provides a theoretical basis for the research of metallurgical triggers.

\section{Conclusion}

In this paper, through the experiment of the low-overload metallurgical tinned silver flake, infers the working mechanism of metallurgical effect, then establishes the mathematical model of metallurgical samples, and through simulation analysis and experiment comparison verification, the following conclusions are finally obtained: 
- The working mechanism of the melt metallurgical effect is: the tin melts and penetrates into the silver layer and forms silver-tin alloy, which causes the thickness of the silver layer to dynamically decrease, the current density continues to increase, and accelerates the temperature rise of the silver layer to its melting point $\left(960^{\circ} \mathrm{C}\right)$. Finally, it makes the tinned silver sample break off quickly;

- In the process of metallurgical effect, the time difference between the tin flux melting, the penetration and the formation of the silver-tin alloy is negligible, and its speed has a linear function relationship with the temperature;

- The error between the simulation and experimental data is within a reasonable range, and the waveform is basically fitted, which can provide a reliable theoretical basis for the study of the metallurgical trigger model.

\section{Reference}

1. Zhou Ting. Simulation Research on Transient Process of High Voltage Current-limiting Fuse[D]. Huazhong University of Science and Technology, 2007.

2. Fu Xue. Design and optimization of arc trigger structure under low overload current[D]. Wuhan: Naval University of Engineering, 2019.

3. Mao Qidong, Shen Bing, Zhuang Jinwu, Dong Runpeng, Hu Xinkai. Optimization of low overload current breaking performance and structural design of DC fuses[J]. High Voltage Technology,2020,46(11):3864-3870.

4. Fu Xue, Qi Lianlian, Zhuang Jinwu, Yan Ling, Lu Wei. Analysis and experimental verification of electrothermal coupling of silver-tinned arc trigger[J]. Electrical Appliances and Energy Efficiency Management Technology,2020(01): 46-50.

5. Zhou Yutao, Zhuang Jinwu, Wu Jin, Zhang Chao, Zhang Li. Analysis and optimization design of gunpowder-assisted breaking breaker[J]. High Voltage Technology, 2020,46(03): 939-946.

6. $\mathrm{Li} \mathrm{Hu}$, Zhou Guangbo. Analysis of the influence of electroplating bath on coating uniformity and coating thickness[J]. Contemporary Research in Chemical Industry, 2020(13):155-156. 